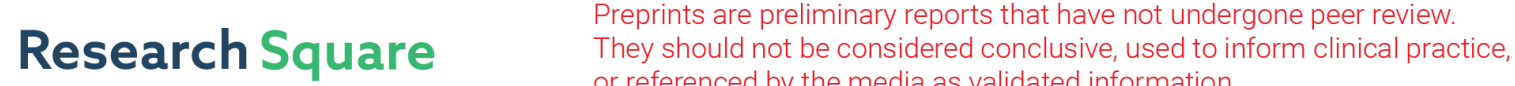 or referenced by the media as validated information. \\ Intelligent Highway Traffic Detection Algorithm based on Deep Learning
}

ming ji ( $\sim$ zhanghappy56@qq.com )

henan provincial department of transportation highway piplline bureau zhengzhou

Chuanxia Sun

Henan Provincial depaprtment of transportation highway ppeline bureau zhengzhou

\section{Yinglei Hu}

Henan Provincial department of transportation highway pipeline bureau zhengzhou

\section{Research Article}

Keywords: Traffic flow prediction, deep learning, intelligent highway, vehicle detection, algorithm

Posted Date: September 27th, 2021

DOI: https://doi.org/10.21203/rs.3.rs-805608/v1

License: (1) This work is licensed under a Creative Commons Attribution 4.0 International License.

Read Full License 


\title{
Intelligent highway traffic detection algorithm based on deep learning
}

\author{
Jin Ming,Sun Chuanxia, Hu Yinglei
}

Henan Provincial Department of Transportation Highway Pipeline Bureau Zhengzhou, China.

Zhanghappy56@qq.com,hnglxxb@163.com,hnglhh@163.com

\begin{abstract}
In order to solve the increasingly serious traffic congestion problem, an intelligent transportation system is widely used in dynamic traffic management, which effectively alleviates traffic congestion and improves road traffic efficiency. With the continuous development of traffic data acquisition technology, it is possible to obtain real-time traffic data in the road network in time. A large amount of traffic information provides a data guarantee for the analysis and prediction of road network traffic state. Based on the deep learning framework, this paper studies the vehicle recognition algorithm and road environment discrimination algorithm, which greatly improves the accuracy of highway vehicle recognition. Collect highway video surveillance images in different environments, establish a complete original database, build a deep learning model of environment discrimination, and train the classification model to realize real-time environment recognition of highway, as the basic condition of vehicle recognition and traffic event discrimination, and provide basic information for vehicle detection model selection. To improve the accuracy of road vehicle detection, the vehicle target labeling and sample preprocessing of different environment samples are carried out. On this basis, the vehicle recognition algorithm is studied, and the vehicle detection algorithm based on weather environment recognition and fast RCNN model is proposed. Then, the performance of the vehicle detection algorithm described in this paper is verified by comparing the detection accuracy differences between different environment dataset models and overall dataset models, different network structures and deep learning methods, and other methods.
\end{abstract}

Key words: Traffic flow prediction, deep learning, intelligent highway, vehicle detection, algorithm

\section{Introduction}

With the continuous development of intelligent transportation systems and the continuous improvement of traffic information collection technology, the collection means and sources of traffic information are increasingly rich. The data collection methods include induction coil detection, radar detection, ultrasonic detection, photoelectric detection, and video detection. The quality and accuracy of the collection are also constantly improved, which makes the cost performance of traffic information collection equipment more and more cost-effective, Therefore, it is possible to obtain real-time traffic data and extract accurate and real-time traffic state information such as traffic flow, speed, and occupancy in the road traffic network. Massive traffic information can provide data support and guarantee for dynamic traffic state analysis and prediction research[1-2].

The purpose of the intelligent transportation system is to improve the efficient and effective information service for people. It mainly includes an advanced traffic information system, advanced traffic management system, advanced vehicle control, and safety system, electronic toll system, and emergency management and rescue system. Traffic guidance technology is the core of an intelligent transportation system, and traffic flow prediction is an important part of traffic guidance technology. How to make full and effective use of massive traffic flow data under the condition of the large road network to make a real-time and accurate prediction of road network traffic conditions is one of the urgent problems in the intelligent transportation system[3].

At present, with the development of information technology, most cities in China have begun to build traffic information integration platforms, including traffic information collection equipment, information transmission equipment, and traffic information release platforms[3-6]. Traffic data management, data processing, and data release need a comprehensive platform to control the real-time update of these dynamic data. The intelligent traffic management system is the center of traffic data management. It can provide traffic information to travelers, provide reference strategies for the choice of traffic routes, and provide travel time, travel services, and other information. Although the current devices used in cities also provide such information, they only release historical traffic information. Because the traffic system is dynamic and complex, it is easy to be interfered with by external conditions. The impact of emergencies or other factors can cause great differences between traffic data. Therefore, the current system equipment does not achieve dynamic real-time information release. Traffic managers and travelers should grasp the changing trend of traffic state in the road network, find out the essential law of traffic flow from a large number of data, actively make decisions, and improve road traffic efficiency. Road network traffic flow prediction is the main basis for traffic management and control departments to take traffic guidance measures. The research on short-term traffic flow prediction models based on road networks has become the focus of traffic flow prediction research in recent years[7-8]. To better reflect the real-time traffic state, with the continuous development of new technologies and the wide application of intelligent systems, higher requirements are put forward for the processing of big data and the accuracy of traffic prediction models. Real-time and accurate traffic flow prediction of the road network can effectively improve the traffic efficiency and safety of the road network, and facilitate the management department to actively carry out traffic planning and take reasonable traffic guidance measures. Therefore, using scientific, reasonable, and effective methods to make an accurate prediction of road network traffic flow is a prerequisite for the development of an intelligent 
transportation system. How to use the existing theoretical system to construct a prediction model and make an effective prediction of large traffic data in road networks has important research significance, and it is also one of the key technologies in road traffic problems.

Traffic flow prediction refers to the prediction of future traffic flow changes based on historical traffic data. The change of traffic flow with time has randomness and is easily disturbed by external conditions. The change of short-term traffic flow is a real-time, non-stationary, non-linear random process. Its change is not only related to the traffic flow conditions of the first few periods of the current monitoring points but also affected by the traffic conditions of the first few moments and the current moment of the upstream and downstream monitoring points. At the same time, the real-time traffic flow will also be affected by weather changes such as rain and snow, sudden changes The impact of various traffic accidents, and traffic environment changes[9].

\section{Research on relevant methods of highway traffic} detection

In the intelligent detection of highway video surveillance, vehicle detection and weather recognition algorithm is the premise of collecting traffic data, and the layout of traffic detector is the basis of practical engineering application. Therefore, this chapter analyzes the main methods of these three technologies, compares the advantages and disadvantages of different methods, and puts forward a suitable detection algorithm and layout model combined with the relevant requirements of highway traffic detection.

2.1 research on environment recognition algorithm

Highway real-time environment recognition is based on the most widely distributed video monitoring equipment, using image processing technology. At present, the mainstream algorithms of environment recognition are: one is to extract the statistical features of the image (usually a variety of features, such as histogram, contrast, etc.) combined with a support vector machine [10-11] (SVM) classifier for recognition; the other is to obtain the sky part image through image segmentation [12-14] and divide it into two parts Thirdly, convolution neural network is used to realize automatic feature extraction and type recognition.

\section{2 environment recognition based on SVM classifier}

Because the weather category is divided into many kinds, such as sunny, cloudy, rainy, and so on, while the traditional SVM classifier can only distinguish the two categories, so the construction of environment recognition algorithm is usually based on a decision tree combined with SVM to distinguish different types of environment. Firstly, the image samples in different environments are collected as the training database, and then the features of each sample are extracted and used as the basis to construct the decision tree, and the SVM classifier is added to the nonleaf nodes of the decision tree to realize the recognition of different environment categories[15-18].

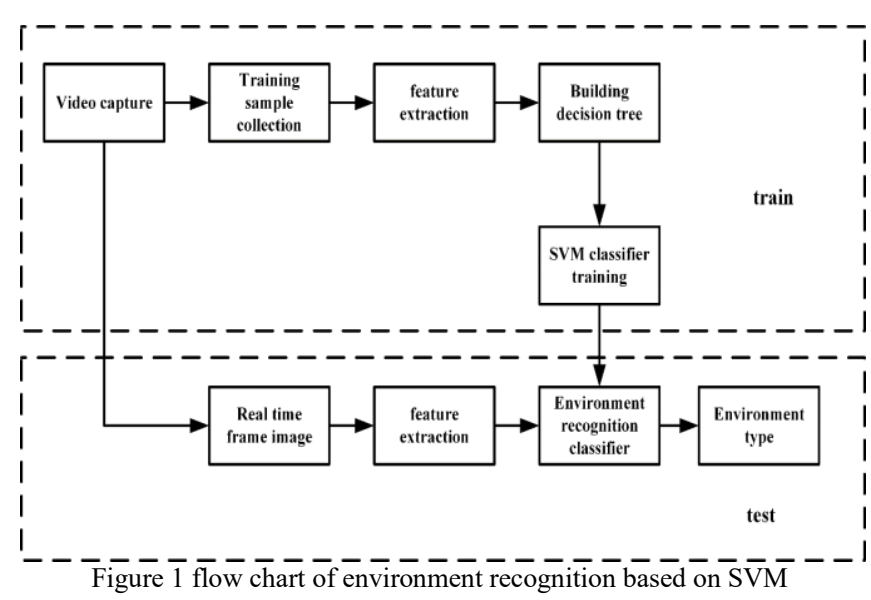

Usually, the image features of the environment are different from those used in other target detection, because they have no fixed region and shape to express. For example, the commonly used gradient histogram (HOG) and local binary pattern (LBP) are not strong in expressing the state of the environment. Therefore, considering the particularity of different environment images, power spectrum slope, contrast, noise, and saturation are often used The results showed that there was no significant difference between the two groups[19-24].

\subsection{1 slope of the power spectrum}

Compared with sunny days, the image is more blurred on rainy days, foggy days, and other weather. We can identify sunny days and non sunny days by comparing the slope of the power spectrum. Firstly, the image is transformed by discrete Fourier transform, and then the power spectrum is calculated

$$
S(u, v)=\frac{1}{M N}|I(u, v)|^{2} \quad(1)
$$

In the above formula, I (U, V) is an image of $\mathrm{n} * \mathrm{~m}$ after discrete Fourier transform, which is usually expressed in polar coordinates

$$
\left\{\begin{array}{l}
(u, v)=(f \sin \theta, f \cos \theta) \\
S(f)=\sum_{\theta} S(f, \theta)
\end{array}\right.
$$

According to the power theory, s (f) decreases exponentially with the increase of $f$

$$
S(f) \approx \frac{A}{f^{\eta}}
$$

Where a is a constant, the power spectrum slope of the image can be obtained from equation (3)

$$
\eta=\ln A-\frac{\ln S(f)}{\ln f}
$$

\subsection{2 contrast characteristics}

Due to the diffraction and refraction of light, the contrast of images in different weather environments is different, so different weather environments can be distinguished. The contrast of the image can be calculated according to the Michaelson formula

$$
C=\frac{L_{\max }-L_{\min }}{L_{\max }+L_{\min }}(5)
$$

In the above formula, $\mathrm{C}$ represents the contrast of the image, minimax, and 11 represent the maximum and minimum pixel intensity values of the image respectively. Also, to improve 
the robustness of the algorithm, the comparison degree can be calculated through the standard deviation of the image intensity

$$
C=\left(\frac{\sum L(x, y)^{2}-\frac{\sum(L(x, y))^{2}}{N}}{N}\right)^{\frac{1}{2}}
$$

\subsection{3 noise characteristics}

On rainy days, snowy days, and other weather conditions, many particles in the air will affect the refraction of light, resulting in different shapes and sizes of noise in the image. Different weather environments can be distinguished by detecting the noise of the image[25-27]. The noise standard deviation of an image can be defined as follows:

$$
\delta=\frac{1}{6(W-2)(H-2)} \sum|L(x, y) \cdot N|
$$

In the above formula, $\mathrm{W}$ and $\mathrm{H}$ are the width and height of the image respectively, $\mathrm{L}(\mathrm{x}, \mathrm{y})$ is the pixel intensity at $(\mathrm{x}, \mathrm{y})$ of the image, and $\mathrm{N}$ is the Laplacian noise prediction template

$$
N=\left(\begin{array}{ccc}
1 & -2 & 1 \\
-2 & 4 & -2 \\
1 & -2 & 1
\end{array}\right) \text { (8) }
$$

\subsection{4 saturation characteristics}

Firstly, the image is converted to HSV color space, and its saturation histogram is counted and normalized as the feature of weather environment recognition.

\subsection{Construction Of SVM Classifier Based On Decision Tree}

To apply SVM to classify different weather environments, the multi-class weather environment is transformed into a multi-level binary classification problem by constructing a decision tree[28-30]. The decision tree is constructed based on the distance of eigenvectors of different weather environments, so the average eigenvectors of different weather environments are calculated first

$$
u_{i}=\frac{1}{\left|X_{i}\right|} \sum_{x \in X_{i}} x
$$

Where IX is the eigenvector of type I weather.

Then the average eigenvectors are normalized, and the Euclidean distance between different eigenvectors is calculated

$$
\left\{\begin{array}{l}
\bar{u}=\frac{1}{N} \sum_{i=1}^{N} u_{i} \\
d_{i j}=\left\|\frac{u_{i}}{\bar{u}}-\frac{u_{j}}{\bar{u}}\right\|
\end{array}\right.
$$

Where $\mathrm{n}$ is the number of weather categories and DIJ is the distance of the eigenvectors between the I and $\mathrm{j}$ categories.

After calculating the distance of different kinds of feature vectors, find the class with the largest distance as a node of the decision tree, and merge other classes as another node. At this time, the former node is a non-leaf node, so an SVM classifier can be constructed to classify the weather category represented by it with other categories. The other node contains multiple weather categories. Continue to build the decision tree according to the above method, and build an SVM classifier in each nonleaf node. Finally, all-weather categories are classified. The diagram is shown in the figure below[31-34].

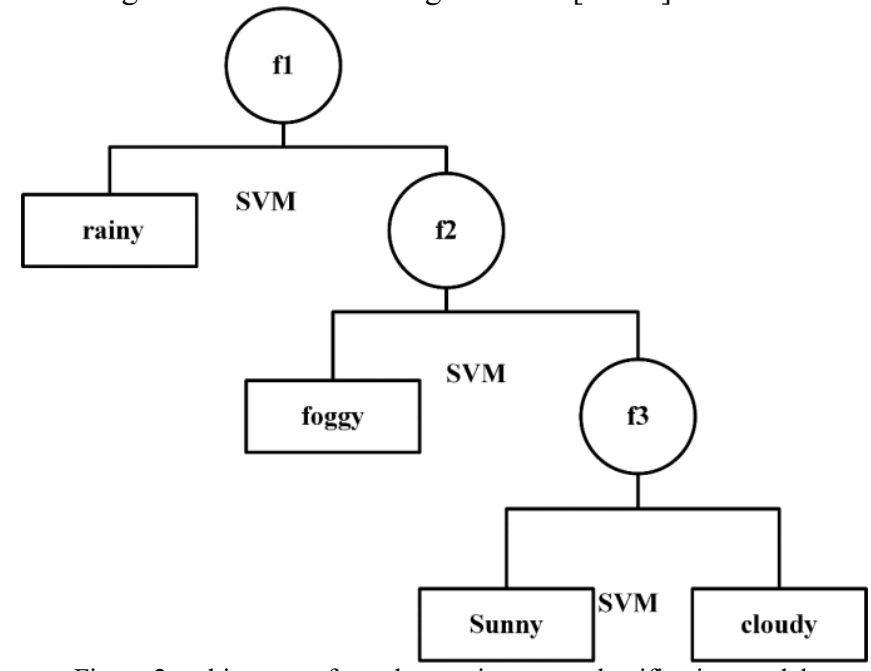

Figure 2 architecture of weather environment classification model For the construction of the SVM classifier, it was initially developed to solve the linear separable problem and find the best hyperplane, which can correctly distinguish two types of targets and maximize the distance between them.

Let the training set be $\left\{\left(x_{1}, y_{1}\right),\left(x_{2}, y_{2}\right), \Lambda,\left(x_{n}, y_{n}\right)\right\}$ and $x \in R^{n}, y \in\{+1,-1\}$, where $\quad x_{i} \quad$ is the sample, $y=+1, y=-1$ represents the different categories of the sample, and $n$ is the sample size, then the hyperplane searched by SVM is the following objective function:

$$
\left\{\begin{array}{l}
\min \frac{1}{2}\|\omega\|^{2} \\
y_{i}\left(\left(\omega \cdot x_{i}\right)+b\right) \geq 1
\end{array}\right.
$$

2.4 Weather Recognition Based On Image Segmentation The weather recognition algorithm based on image segmentation first extracts the sky part of the image to propose the interference of other parts of the image, then selects the appropriate features to describe different weather environments based on multi-core learning, and finally judges the weather type through SVM classifier. The algorithm flow is shown in the figure below.

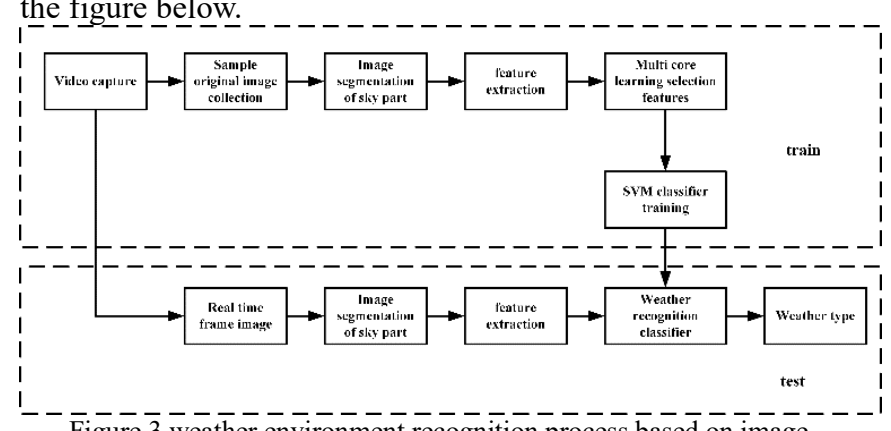

Figure 3 weather environment recognition process based on image segmentation

\subsubsection{Sky Image Segmentation}

The recognition of the sky in the image is based on the fact that the sky is above the ground and the cloud is moving relative to other objects. First of all, due to the mobility of clouds, we can calculate the accumulated residuals of multiple consecutive images. Also, since the sky is at the top of the 
image, we can give different weights to the residuals of different positions to reduce the influence of moving objects and reflected light, that is, we can give greater weight to the higher residuals.

Table 1 pseudo-code of sky image segmentation algorithm 1: Initialization weight: proportional to the square of height

\section{2: Last $=$ first frame, residual $=0$, count $=0$}

3: Threshold $=\mathrm{m}$, update interval period $=\mathrm{n}$

4: When to read a new image

5: Residual=Residual abs(current-last)*Weig

6: Mask normalize ( Residual) $>$ Threshold

7: Close and open the mask

8: if Count Penod== 1 then

9: Residual Residual* Mask

10: end if

11: Count++

12:Last s Current

13: end while

14: Output sky part image mask

2.4.2 Construction Of Classifier Based On Multi-Kernel Learning

Firstly, the sky image features are extracted, which usually include the following five types of features

(1) The histogram features of three channels in HSV color space are the description of image color;

(2) Photo: a scale-invariant feature descriptor with a fixed size to represent the shape of the image;

(3) LBP: local binary pattern feature, which represents the texture of the image;

(4) Gradient: gradient feature, the image gradient calculated by Sobel operator represents the texture of the image;

(5) Motion: motion feature, obtained by calculating the residuals of consecutive frames, characterizes the operation characteristics of the image.

Conventional SVM in the learning algorithm, a variety of features are simply fused, but different features have different contribution rates to weather classification. Simple feature fusion will weaken the utility of important features and reduce the classification results. Also, for complex image feature classification, which is a nonlinear separable problem, we need to apply kernel function to map it to high-dimensional space for classification the choice of number is also a problem to be solved. The multi-kernel learning method can weigh different features according to their contribution rate. Its principle is to perform kernel operation on each feature vector corresponding to a kernel function, fuse different kernel functions through linear combination, and add Lagrange multipliers to them, and transform them into dual problems to solve. Finally, different weights corresponding to each feature and its kernel function are obtained, and the algorithm flow is optimized the diagram is as follows.

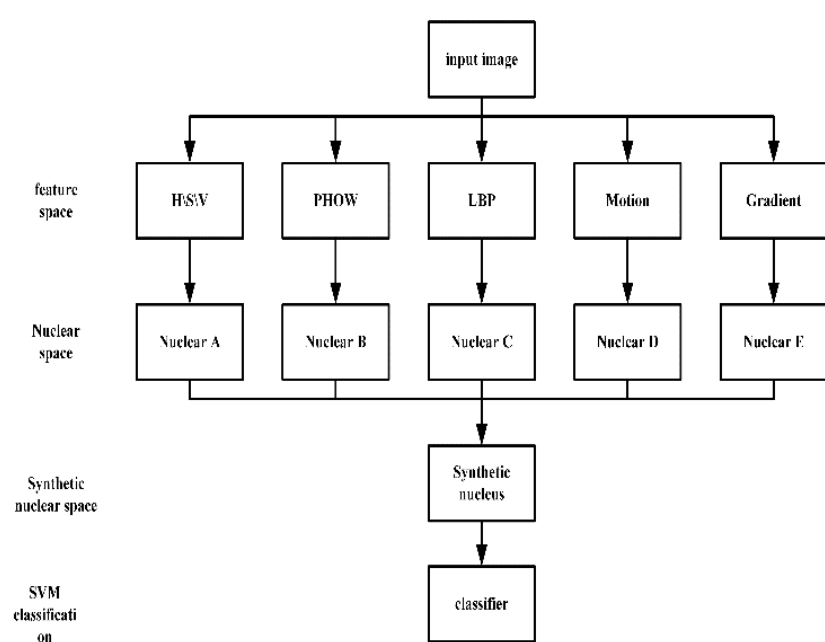

Figure 4 flow chart of the multi-core learning algorithm In solving SVM classifier based on multi-kernel learning, the basic principle is the same as that of ordinary single kernel SVM, but formula (12) is transformed into a linear combination of several different kernel functions when calculating the composite kernel

$$
k\left(x, x_{i}\right)=\sum_{m-1}^{M} d_{m} k_{m}\left(x, x_{i}\right)
$$

Where $\mathrm{m}$ is the number of nuclei, $\mathrm{m} \kappa$ is the different kernel function corresponding to different characteristics, DM is the linear combination coefficient of different nuclei, which satisfies $\square 0 \mathrm{MD}$ and has mmmd11. Similarly, according to the KKT condition, using the Lagrange multiplier, the objective function (13) can be rewritten as the following dual problem:

$$
\left\{\begin{array}{l}
\max \left(\sum_{i=1}^{n} \lambda_{i}-\frac{1}{2} \sum_{i, j=1}^{n} \lambda_{i} \lambda_{j} y_{i} y_{j} \sum_{m-1}^{M} d_{m} k_{m}\left(x_{i}, y_{i}\right)\right) \\
s, t: \sum_{i=1^{\prime}}^{n} \lambda_{i} y_{i}=0, \lambda_{i} \geq 0(i=1,2, \Lambda, n) \\
\sum_{m=1}^{M} d_{m}=1
\end{array}\right.
$$

Finally, the dual problem can also be solved by the SMO algorithm to obtain the classifier.

\subsection{Weather Recognition Based On Deep Learning}

The weather recognition method based on deep learning makes full use of the powerful expression ability of deep learning networks and applies a convolution neural network to automatically extract the features of different weather states and classify them. Firstly, a large number of samples of different weather conditions are collected and preprocessed by image size transformation and pixel centralization; secondly, the sample features are extracted by convolution neural network, and the neural network parameters of this part are obtained based on error backpropagation training; finally, the weather category of the image is determined by a softmax layer. The flow chart of the weather type recognition algorithm based on a convolution neural network is shown in the figure below. 


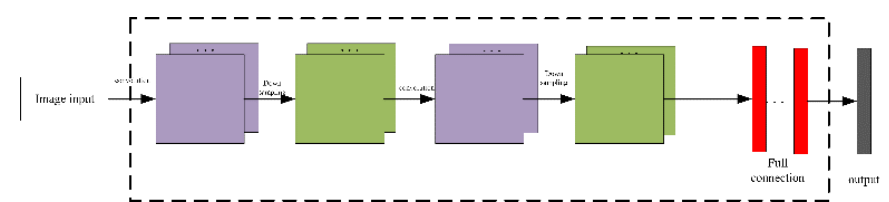

Figure 5 weather recognition process based on deep learning 3 Research on the vehicle detection algorithm

The main purpose of the highway vehicle detection algorithm is to collect traffic flow data through a video monitoring system based on image processing. The mainstream algorithms include background subtraction [35], detection algorithm based on artificial feature [36] combined with classifier, and vehicle detection algorithm based on deep learning [37]. This section mainly describes the principles of these three algorithms.

3.1 Vehicle Detection Algorithm Based On Background Difference

Background subtraction algorithm is one of the earliest image sequence target recognition algorithms. Its core idea is to establish a complete background model, calculate the difference between the real-time frame image and the background model to extract the foreground target. The algorithm flow is shown in Figure 6.

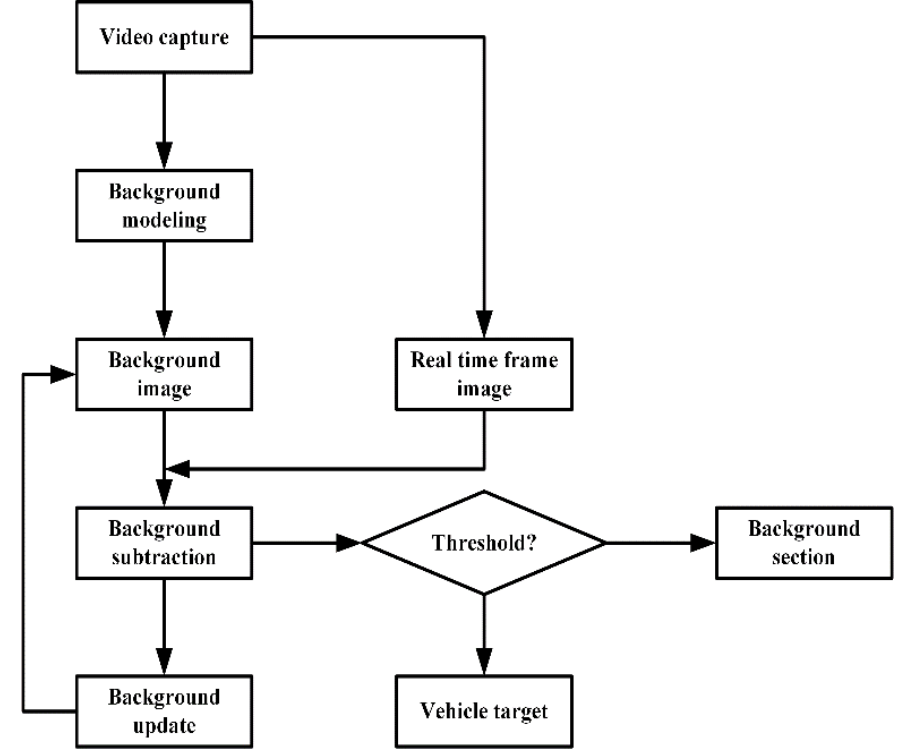

Figure 6 flow chart of vehicle recognition algorithm based on background difference

The key point of the background subtraction algorithm lies in the background modeling and the determination of the difference threshold. Firstly, the background model on the one hand requires that it can not contain foreground objects to ensure that the different results can detect vehicles, on the other hand, it requires that the background can be updated in time to avoid a lot of noise after the difference. Background models mainly include the mean model [18], Gaussian model [20], and Gaussian mixture model [31]. Different algorithms have their advantages and disadvantages. The following two modeling methods are discussed.

The average background modeling method considers that the influence of moving target can be eliminated by superposing continuous multi-frame images and seeking the average value, to build a clean background model. This is because the vehicle is a fast-moving target and its duration in the image is very short. Therefore, the pixels of the vehicle can be averaged by superposing continuous multi-frame images, to obtain the pixel value close to the background. The time series means the model is widely used in the average background method:

$$
B G_{t}=\frac{1}{N} \sum_{i=1}^{N} x_{i} \quad \text { (14) }
$$

Where BGT is the background image at time $t, n$ is the number of consecutive image frames before time $t$ used to establish the background model, and IX is the i-th frame of the image. Generally, taking about 1000 frames of $\mathrm{N}$ can get a better clean background. After obtaining the background image, there are different ways to update the background:

$$
B G_{t+1}=(1-\alpha) B G_{t}+\alpha \times \text { Frame (15) }
$$

$\mathrm{BG} \mathrm{T}+1$ is the background image at $\mathrm{t}+1, \alpha$ is the fixed learning rate, and the frame is the newly acquired image frame. In this updating method, the learning rate is one of the most important parameters. If the learning rate is too low, the background will not be updated in time, resulting in more noise points when the current frame has differed from the background image, and the vehicle target cannot be detected accurately; if the learning rate is too high, it is easy to add the foreground target to the background, so that the background image contains the vehicle target, which also affects the poor performance the effect of points. Therefore, the learning rate needs to determine a reasonable value according to the changing speed of the environment, and often this value can not be uniquely determined in the outdoor environment, which becomes the constraint of this method.

Also, the background can be updated at one time after a fixed interval. Suppose that after T1, the system obtains $m$ frames of new images:

$$
B G_{n e w}=\frac{1}{N}\left(\sum_{i=1}^{N-M} x_{i}+\sum_{i=1}^{M} x_{j}\right)
$$

In the above formula, Agnew is the updated model, which is established by a group of new image sets composed of the last $\mathrm{N}-\mathrm{M}$ frames in the N-frame images constituting the previous background image and the M-FRAME images acquired subsequently, that is, the background is recalculated with the $\mathrm{N}$-frame images closest to the current time.

3.2 vehicle detection algorithm based on statistical learning The vehicle detection algorithm based on statistical learning rises from the establishment of manual features. This kind of method breaks through the limitation that background difference only detects foreground objects which are different from the background, and starts the real vehicle target detection. Directional gradient histogram feature (HOG), local binary pattern feature (LBP), and Haar feature are the most widely used features in statistical learning methods, especially the first two, which have strong expression ability and can well distinguish vehicle targets in the image, so they are often used in highway intelligent traffic system. Because the principles of vehicle detection algorithms using different features are similar, this paper introduces the process of vehicle detection based on the fusion of hog and LBP features.

The vehicle detection algorithm based on statistical learning first needs to collect a large number of vehicle samples and nonvehicle samples in different environments, count the hog and LBP features of each sample, fuse the feature vector and use a support vector machine (SVM) to train the classification of the vehicle and nonvehicle samples, and detect the vehicle target based on the trained classifier. The algorithm process is 
shown in the figure below.

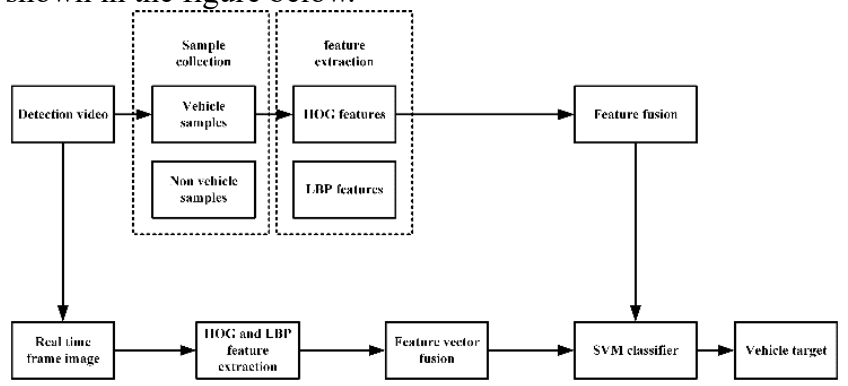

Figure 7 vehicle detection algorithm flow based on fusion feature

\section{Traffic detection based on deep learning}

Highway video monitoring can collect the traffic parameters needed for traffic flow state analysis, including flow, speed, occupancy, headway, vehicle type and its ratio, traffic flow density, etc. This paper studies the data collection of flow, speed, occupancy, vehicle type, and its ratio based on deep learning vehicle detection algorithm.

\subsection{Speed Detection}

The principle of vehicle speed detection based on video monitoring is to mark a fixed-length virtual detection area on the monitoring image and determine its speed by detecting the time of vehicle passing through the detection area.

When the time of the vehicle passing through the start L1 of the detection area is T1 and the time of passing through the end $\mathrm{L} 2$ is $\mathrm{T} 2$, the speed can be calculated as follows:

$$
v=\frac{d}{T 2-T 1}(17)
$$

To ensure the accuracy of speed detection, the time of a vehicle passing through the virtual detection area is the time when the video monitoring first detects the beginning and end of vehicle contact. Also, the length $\mathrm{d}$ of the virtual area needs to correspond to the actual distance, so the selection of the virtual detection area needs to be based on the road markings. According to the standard length of the road markings, the length of the middle white line is 6 meters, and the interval is 9 meters. Then the vehicle speed can be accurately calculated according to equation (18).

\section{2 flow detection}

Compared with other detection algorithms, vehicle detection based on deep learning has higher accuracy, which can realize vehicle recognition of different distances and scales. Therefore, the number of vehicles in each frame can not be simply superimposed for traffic statistics. The number of vehicles accurately detected by the system for the monitoring image is 4 . If the number of vehicles in each frame is superimposed, it will greatly increase the statistical number of vehicles and make the parameters invalid. According to the virtual detection area, the traffic flow statistics can be defined as the number of vehicles passing through the detection area, that is, when the vehicles pass through the two ends of the detection area, the traffic flow statistics is carried out, while for other vehicles that do not pass through the detection area, the traffic flow parameters can be obtained after the traffic flow detection of unit time t. Through the statistical method of the virtual detection area, the vehicle speed can be calculated while the traffic flow is counted, which makes the algorithm realize the collection of multiple parameters and reduce the additional amount of calculation. 4.3 occupancy detection
The road occupancy rate refers to the ratio of the cumulative time of vehicles passing through any road section to the total observation time. Assuming that the unit time of video monitoring observation is $t$, and the cumulative time of vehicle detection based on deep learning is $t$, the road occupancy rate of the video detection area can be obtained as follows:

$$
p=\left(\frac{t}{T} \times 100\right) \%
$$

Similarly, because the parameter detection of flow and speed needs vehicle identification, only the time of detecting vehicle target is counted at the same time to calculate the vehicle occupancy of the road, and the detection process does not need additional calculation.

\section{4 vehicle type and its ratio detection}

A prominent advantage of a road vehicle detection algorithm based on deep learning is that it can identify different vehicle types. In this paper, vehicle types are divided into five categories: car, minibus, bus, truck, and trailer. At the same time of vehicle detection, the vehicle type of each vehicle target can be obtained, assuming that the statistical value of traffic volume per unit time is $n$, while the statistical value of the I vehicle type is $n \mathrm{n}$. Then the traffic volume ratio of class I vehicles is:

$$
w=\left(\frac{n}{N} \times 100\right) \%
$$

The statistics of vehicle type ratio is calculated based on the statistics of traffic volume, and the vehicle detection based on deep learning can recognize the vehicle type while recognizing the vehicle target, so the calculation of vehicle type ratio can be completed at the same time of traffic volume statistics.

\section{Traffic incident detection based on deep learning}

Compared with other traffic detectors, video monitoring can not only collect road traffic parameters, but also detect traffic events through video images, providing traffic managers with effective road traffic conditions for corresponding efficient traffic control, timely handling traffic accidents, avoiding the second occurrence of traffic accidents, ensuring the life safety of users, and maintaining the smooth road function. In this paper, the road environment is identified based on a deep learning algorithm. On this basis, the corresponding vehicle detection model is applied to realize the collection of traffic parameters, and the automatic detection of road traffic events is studied by using the statistical traffic parameter information. Under the normal driving state of the highway, the vehicle is running at high speed. If there is parking, an abnormal event likely occurs, which makes the vehicle stop in the middle of the road or the emergency lane. Therefore, through the intelligent identification of parking events by the video monitoring system, the traffic accidents on the highway can be found at the first time, and the corresponding countermeasures can be taken according to the real-time environmental conditions and traffic flow status Emergency rescue measures, efficient handling of traffic accidents, keep the road unobstructed.

The deep learning model is used to detect the vehicle target and its location accurately, and the parking event is judged by counting the continuous multiple frames of video images in the same location. To ensure the accuracy and robustness of the algorithm, in the process of parking event detection, it is 
necessary to effectively select the continuous observation time and the time when there is a vehicle target at the same location within the observation time. If the continuous observation time is too long, the real-time performance of the algorithm will be reduced, and the detection speed of the event will be delayed. If the continuous observation time is too short, it is easy to misjudge. Similarly, the same location is the same the selection of the time threshold of the vehicle target also affects the real-time performance and robustness of the algorithm.

\section{Conclusion}

Based on the deep learning method, this paper carries out highway environment recognition and vehicle detection realizes the accurate and efficient traffic flow parameter collection and traffic event detection and provides an effective data basis for the traffic operation state analysis. The main research contents and achievements study several commonly used weather recognition algorithms based on video image processing and analyze the weather recognition algorithm based on decision tree and SVM The principles of classification method, image segmentation combined with SVM classification method and weather environment type recognition based on deep learning method are introduced. The performance differences between different algorithms are compared, and the framework of the highway weather environment recognition model based on a deep neural network is proposed.

\section{Ethical approval}

My paper does not deal with any ethical problems.

\section{Funding details}

This work was supported by Research on big data application technology of smart highway (No: 2016Y4) and complete technology of highway video monitoring and perception based on big data analysis (No: 2019G1)

\section{Conflict of interest}

The authors declared that they have no conflicts of interest to this work.

\section{Informed Consent}

We declare that All authors have informed Consent.

\section{References}

[1] Zhong Xinhao, long Yonghong, he zhenkai, Li Peiyun. Helmet wearing detection based on improved tiny yolov3 algorithm [J]. Journal of Hunan University of Technology, 2021,35 (02): 46-50

[2] Yang Yahu, Wang Yu, Chen Tianhua. Remote video surveillance abnormal image detection based on deep learning [J]. Telecommunication technology, 2021,61 (02): 203-210

[3] Kang Shuo, Ke Zhenzheng, Wang Xuan, Zhu Weidong. Detection method of wire laying defects based on infrared and visible image fusion [J / OL]. Acta Aeronautica Sinica: 1-13 [2021-02-24]

[4] Fang Kai. Fence crossing behavior detection method based on deep learning [J]. Computer system application, 2021,30 (02): 147-153

[5] Xu Jiayu, Lin Chuye, Chen Zhitao, Deng zhuoran, pan Jiahui, Liang Yan. Handwritten calligraphy font recognition algorithm based on deep learning [J]. Computer system applications, 2021,30 (02): 213-218

[6] Su Yang, Lu Xiang, Li Kun, Zhang shaochao. Research on character detection in computer room based on lightweight deep learning network [J]. Industrial instrumentation and automation, 2021 (01): $100-103$

[7] Zhang Chen Yang, Huang Teng, Wu Zhuang Zhuang. Rgb-d slam method based on K-means and deep learning [J / OL]. Computer Engineering: 1-14 [2021-02-24]

[8] Xu Degang, Wang Lu, Li Fan. Review of typical target detection algorithms based on deep learning [J / OL]. Computer engineering and application: 1-21 [2021-02-24]

[9] Chen Changchuan, Wang Haining, Zhao Yue, Wang Yanping, Li
Lianjie, Li Kui, Zhang Tianqi. A new traffic sign recognition algorithm based on deep learning [J]. Telecommunication technology, 2021,61 (01): 76-82

[10] Cao Yangyang, Luo Yinsheng, Liu Yadong, Song Wei, Chen Chuanyi. Design of navigation system for plant protection machine based on slam and deep learning [J]. Industrial control computer, 2021,34 (01): 73-76

[11] Jiang Shan, sun yuan, Yan daosen. Insulator recognition of aerial inspection image based on deep learning algorithm [J]. Journal of Fuzhou University (NATURAL SCIENCE EDITION), 2021,49 (01): $58-64$

[12] Wu Xue, song Xiaoru, Gao Song, Chen Chaobo. Overview of target detection algorithm based on deep learning [J]. Sensors and Microsystems, 2021,40 (02): 4-7 + 18

[13] Liu Yu Yu, Ma Jian Xiao, Wang Yu Chen, Bai Ying Jia, Xie Zheng Jun. pedestrian detection algorithm in forest area based on improved cornernet Lite [J / OL]. Journal of Forestry Engineering: 1-6 [2021-02-24]

[14] Dang Shunfeng, Xiong Rui, Li Jihui, Chen Canqi, Chen Zhenwei, Wu Xin. Parking space detection based on improved mask RCNN algorithm [J]. Modern manufacturing engineering, 2021 (01): 91-97 + 101

[15] Li Hua, Wang Yanbin, Yi Peng, Wang Teng, Wang Changliang. Research on hard hat recognition in complex work scenes based on deep learning $[\mathrm{J}]$. China Science and technology of work safety, 2021,17 (01): 175-181

[16] Huang Shengpeng, fan Pingqing. Research on real time vehicle detection based on deep learning [J]. Software engineering, 2021,24 (01): $13-16+5$

[17] Zhao Qihui, Liu Yanyang, Xiang Yanping. Overview of single stage vehicle detection algorithm based on deep learning [J]. Computer applications, 2020,40 (S2): 30-36

[18] Nan Xiaohu, Ding Lei. Survey of typical target detection algorithms based on deep learning [J]. Computer application research, 2020,37 (S2): 15-21

[19] Liu Yang, Zhan yinwei. Survey of small target detection algorithm based on deep learning [J]. Computer engineering and application, 2021,57 (02): 37-48

[20] Liu Teng, Shang Shaowei, Ge Fu, song Yiqing, Liu Hongzhe. Improved vehicle detection method based on centernet [a]. Network application branch of China Computer Users Association. Proceedings of the 24th Annual Meeting of network new technology and application of China Computer Users Association in 2020 [C]. Network application branch of China Computer Users Association: Beijing Key Laboratory of information service engineering, Beijing Union University, 2020:7

[21] Weixing, Yang Guoqiang, Lu Yang, Wei Zhen. Vehicle sequential motion detection algorithm based on deep learning [J]. Computer engineering and design, 2020,41 (12): 3510-3516

[22] Wang Na. Research on pedestrian detection algorithm and its safety in unmanned driving [D]. Nanjing University of Posts and telecommunications, 2020

[23] hang Jingjing. Pedestrian self occlusion detection and application based on deep learning [D]. Nanjing University of Posts and telecommunications, 2020

[24] Guo Chuang. Research on text detection in natural scenes based on deep learning [D]. Nanjing University of Posts and telecommunications, 2020

[25] Shi Jianwei. Design and implementation of license plate recognition system based on deep learning [D]. Nanjing University of Posts and telecommunications, 2020

[26] yuan Zhuzhu. Pedestrian detection and counting based on deep learning [D]. Nanjing University of Posts and telecommunications, 2020

[27] Fu Ning. Research on scene structural description method based on deep learning [D]. Nanjing University of Posts and telecommunications, 2020

[28] Qiu Xuyang, Huang Yingping, Guo Zhiyang, Hu Xing. Obstacle detection and depth estimation based on deep learning [J]. Journal of Shanghai University of technology, 2020,42 (06): 558-565

[29] Li Kejian, Chen Shaobo, Li Wanqi. Simulation of traffic sign detection system based on deep learning [J]. Software guide, 2020,19 (09): 31-34 
[30] Zhang Peipei, Wang Zhao, Wang Fei. Research on image target detection algorithm based on deep learning [J]. Foreign electronic measurement technology, 2020,39 (08): 34-39

[31] Shi Jianwei. Design and implementation of license plate recognition system based on deep learning [D]. Nanjing University of Posts and telecommunications, 2020

[32] yuan Zhuzhu. Pedestrian detection and counting based on deep learning [D]. Nanjing University of Posts and telecommunications, 2020

[33] Fu Ning. Research on scene structural description method based on deep learning [D]. Nanjing University of Posts and telecommunications, 2020

[34] Zhang Shulin. Research on target detection algorithm based on deep learning [D]. Guangdong University of technology, 2020

[35] Wang junqun, Li Huapeng. Intelligent analysis of vehicle violations based on deep learning algorithm [J]. Journal of Shanghai Institute of shipping and Transportation Sciences, 2019,42 (04): 49-54

[36] Fang Yong, Dang Qian, Wan Jian, Wang Weifeng, Chen Aiwei. Road traffic congestion detection based on deep learning [J]. Intelligent City, 2018,4 (23): 1-3

[37] Lu Xue, Liu Kun, Cheng Yongxiang. A deep learning algorithm for non motor vehicle target detection [J]. Computer engineering and applications, 2019,55 (08): 182-188 + 214 